\title{
Connectivity and Critical Currents in Polycrystalline $\mathrm{MgB}_{2}$
}

\author{
M Eisterer, J Emhofer, S Sorta, M Zehetmayer, H W Weber \\ Atominstitut der Österreichischen Universitäten, \\ Vienna University of Technology, 1020 Vienna, Austria
}

\begin{abstract}
Current transport in polycrystalline magnesium diboride is highly non-uniform (percolative) due to the presence of secondary phases and also due to the intrinsic anisotropy of the material. The influence of secondary phases on the transport properties of $\mathrm{MgB}_{2}$ was investigated. Bulk samples were prepared from a mixture of $\mathrm{MgB}_{2}$ and $\mathrm{MgO}$ powders by the ex-situ technique in order to vary the $\mathrm{MgO}$ content systematically. The samples were characterized by resistive and magnetization measurements. The reduced $\mathrm{MgB}_{2}$ fraction is modeled by a reduced effective cross section (connectivity), which was assessed directly by the experiments. The presence of $\mathrm{MgO}$ also increases the percolation threshold, which reduces the zero resistivity (or irreversibility) field.
\end{abstract}




\section{INTRODUCTION}

Magnesium diboride is an interesting material for applications. It is cheap and can potentially be operated at higher temperatures than the conventional superconductors NbTi or $\mathrm{Nb}_{3} \mathrm{Sn}$. Unfortunately, the upper critical field, $B_{c 2}$, of pure $\mathrm{MgB}_{2}$ is comparatively small ( 14 T at $0 \mathrm{~K}[1])$ and the upper critical field anisotropy [1] leads to a strong magnetic field dependence of the critical current density, $J_{\mathrm{c}}$ [2]. Thus the in-field performance of clean $\mathrm{MgB}_{2}$ is modest, but can be improved by the introduction of small impurities, which act as scattering centers, reduce the mean free path of the charge carriers and, therefore, enhance $B_{c 2}$. Today's best wires already touch the performance of NbTi [3, 4]. It was shown in Ref. [1], that the critical currents in $\mathrm{MgB}_{2}$ are still smaller by a factor of about five compared to expectations for an optimized material. It is non trivial to decide whether this difference is caused by comparatively weak pinning (compared to optimum) or by a reduced cross section over which the currents flow [5], but it seems that the latter is more important [1]. In fact, the density of the superconducting filaments in typical wires or tapes is often only around half the theoretical one and secondary phases, especially oxides [6, 7, 8] or boron rich compounds [8], are found at grain boundaries. The latter are expected to block the current flow. The corresponding reduction in cross section is usually called reduced area or connectivity problem and can be quantified by a factor $A_{\text {con }} \leq 1$. The current has to meander between the well connected grains and this current percolation is amplified by the upper critical field anisotropy of $\mathrm{MgB}_{2}$, when a magnetic field is applied. The grains of the current carrying "backbone" attain different properties according to their crystallographic orientation with respect to the applied field. A model for the current flow in $\mathrm{MgB}_{2}$ was

proposed in Ref. [2]. It is based on the introduction of an effective cross section $A_{p} \sigma_{0}$ (similar to $A_{\text {con }}$ ) as a function of the fraction of superconducting grains, $p$, which changes with field, temperature, and applied current. $\sigma_{0}$ denotes the geometrical cross section, thus $A_{p}$ varies between 0 and 1 . The original model assumed in principle that all grains (or sites in the terminology of percolation theory) consist of $\mathrm{MgB}_{2}$ and are perfectly connected. If these assumptions become invalid, adoptions have to be made, which depend on the actual defect structure, as discussed in the following paragraphs.

Each non-superconducting inclusion (or pore) reduces $A_{\text {con }}$, which becomes an additional parameter of the model [1]. The percolation threshold $p_{\mathrm{c}}$ (defined as the minimum fraction 
of superconducting grains which is necessary for a continuous current path) is expected to change only if the average number of connections between neighbouring (superconducting) grains is reduced. The number of connections between nearest neighbors is constant in a regular lattice and called coordination number, $K$. The percolation threshold is closely related to the coordination number [9, 10], the larger $K$, the smaller is $p_{\mathrm{c}}$.

An instructive and simple situation occurs, if the morphology of the defects is comparable to the morphology of the $\mathrm{MgB}_{2}$ grains. The structure of the system does not change, but the number of superconducting grains is reduced to $p_{\mathrm{MgB}_{2}} p . p_{\mathrm{MgB}_{2}}$ is the fraction of $\mathrm{MgB}_{2}$ grains and $p$ denotes further on the superconducting fraction among the $\mathrm{MgB}_{2}$ grains (which depends on temperature, field, and current). If $A_{p}$ is a simple power law of the form

$$
A_{p}\left(p, p_{c}\right)=\left(\frac{p-p_{c}}{1-p_{c}}\right)^{t}
$$

it can be rewritten as [11]

$$
\begin{aligned}
A_{p}\left(p_{\mathrm{MgB}_{2}} p, p_{c}\right) & =\left(\frac{p_{\mathrm{MgB}_{2}} p-p_{c}}{1-p_{c}}\right)^{t} \\
& =\left(\frac{p_{\mathrm{MgB}_{2}}\left(p-p_{c} / p_{\mathrm{MgB}_{2}}\right)}{1-p_{c}}\right)^{t} \\
& =\left(\frac{p_{\mathrm{MgB}_{2}}\left(p-p_{c}^{*}\right)}{1-p_{c}^{*}} \frac{1-p_{c}^{*}}{1-p_{c}}\right)^{t} \\
& =\left(\frac{p-p_{c}^{*}}{1-p_{c}^{*}} \frac{p_{\mathrm{MgB}_{2}}\left(1-p_{c}^{*}\right)}{1-p_{c}}\right)^{t} \\
& =\left(\frac{p-p_{c}^{*}}{1-p_{c}^{*}}\right)^{t}\left(\frac{p_{\mathrm{MgB}_{2}}-p_{c}}{1-p_{c}}\right)^{t} \\
& =A_{p}\left(p, p_{c}^{*}\right) A_{p}\left(p_{\mathrm{MgB}_{2}}, p_{c}\right) \\
& =A_{p}\left(p, p_{c}^{*}\right) A_{\mathrm{con} .}
\end{aligned}
$$

Thus, $A_{p}\left(p_{\mathrm{MgB}_{2}}, p_{c}\right)$ splits into a field and temperature independent factor, which is just the connectivity, $A_{\text {con }}$, and a modified $A_{p}$ needed for the percolation model [2]. $A_{\text {con }}$ is given by the same power law as the original $A_{p}\left(p, p_{c}\right)$, with $p$ equal to $p_{\mathrm{MgB}_{2}}$. The percolation threshold in the modified $A_{p}$ increases to $p_{c}^{*}=p_{c} / p_{\mathrm{MgB}_{2}}$. This increase reduces the zero resistivity field and increases the field dependence of the critical currents [12]. Note that the increase of the percolation threshold is independent of the actual function of $A_{p}$, since it just results from the condition that the total fraction of superconducting grains, which is reduced by $p_{\mathrm{MgB}_{2}}$, still has to be $p_{c}$. 
The same scenario occurs if individual $\mathrm{MgB}_{2}$ grains are completely covered by an insulating layer and disconnected from the connected matrix, $p_{\mathrm{MgB}_{2}}$ then refers to the fraction of connected $\mathrm{MgB}_{2}$ grains.

If the insulating inclusions (the same holds for pores) and their separation are much larger than the superconducting grains, mainly $A_{\text {con }}$ is reduced, since the average coordination number does not change significantly. Only grains neighboring these non-superconducting sites, have less connections to $\mathrm{MgB}_{2}$ grains, but their number is small, since the distance between the inclusions is assumed to be much larger than the diameter of the $\mathrm{MgB}_{2}$ grains. The reduction in $A_{\text {con }}$ is expected to have a similar dependence on the non superconducting volume fraction for both small and large inclusions.

If the inclusions are much smaller than the $\mathrm{MgB}_{2}$ grains, or if they have a totally different morphology, it is hardest to predict their influence. The blockage of individual connections (not whole grains) by insulating phases is expected to result in an increase of the percolation threshold (reduction of the average coordination number) and a reduction in $A_{\text {con }}$. Since the insulating layers might be much thinner than the diameter of the $\mathrm{MgB}_{2}$ grains, a small volume fraction of secondary phases might result in a strong effect. The problem changes from site to bond percolation [13] and the volume fraction is not the ideal parameter in this case. In principle one expects a similar dependence of $A_{\text {con }}$ on the fraction of conducting bonds, although with a different percolation threshold. (It becomes a mixed bond/site percolation problem, when a magnetic field is applied). However, the fraction of conducting bonds is difficult to asses in real systems, but $p_{c}$ of the site percolation problem might increase, because of a reduction in the average coordination number of the $\mathrm{MgB}_{2}$ matrix.

Small inclusions (compared to the $\mathrm{MgB}_{2}$ grain size) are not expected to influence $p_{\mathrm{c}}$, if they do not block whole grain boundaries, but only reduce the conducting area between the grains, or, if they are incorporated into the $\mathrm{MgB}_{2}$ grains. The reduction in $A_{\text {con }}$ might be (over-)compensated by the potential pinning capability of small defects. If these small inclusions block whole connections, because they cluster at individual grain boundaries, their influence should be similar to insulating layers.

Although the reduction of $J_{c}$ by poor connectivity is quite obvious [5], 14], only a few studies were aiming at a quantitative relationship between the amount of secondary phases or pores and the corresponding reduction of the critical currents so far [15, 16, 17]. In this work, the non-superconducting volume fraction is changed by $\mathrm{MgO}$ particles, which 
were added to the $\mathrm{MgB}_{2}$ powders before sintering. The influence of the decreasing $\mathrm{MgB}_{2}$ volume fraction on the resistivity and on the superconducting properties is investigated and described by changes in $p_{\mathrm{c}}$ and $A_{p}$.

\section{EXPERIMENTAL}

Powders of $\mathrm{MgB}_{2}$ (Alfa Aesar, 98\%, -325 Mesh) and MgO (Alfa Aesar, 99.95\%, $325 \mathrm{Mesh}$ ), were weighed, mixed, and pressed uniaxially with $750 \mathrm{MPa}$. The nominal MgO content ranged from 0 to $60 \mathrm{vol} \%$. The pressed pellets had a diameter of $13 \mathrm{~mm}$ and a thickness of around $2.6 \mathrm{~mm}$. Each sample was placed into an iron container and sintered separately in argon atmosphere for $10 \mathrm{~h}$ at $1035^{\circ} \mathrm{C}$. The temperature was ramped up with $500^{\circ} \mathrm{C} / \mathrm{h}$. The sample cooled down slowly after the heat treatment (within a few hours). The iron container was necessary to prevent magnesium loss, which was monitored by comparing the weight of the pellet prior to and after sintering. A stripe (typically $10 \times 1.6 \times 1.5 \mathrm{~mm}^{3}$ ) was cut from the central part of the sample for the resistive measurements. A slice (around $2 \times 1.5 \times 0.4 \mathrm{~mm}^{3}$ ) was prepared from the central part of the stripe after the resistive measurements for the assessment of the critical current density.

The nominal volume fraction of $\mathrm{MgB}_{2}$ was calculated from the weight and the theoretical density of the mixed powders. Since the density of the samples was only around $70 \%$ of the theoretical density, this nominal volume fraction strongly overestimates the real volume fraction. Therefore, this quantity is denoted as nominal content in the following. A better estimate of the real volume fraction was calculated from the weight of the admixed $\mathrm{MgB}_{2}$ powder, the theoretical density of $\mathrm{MgB}_{2}$ and the final volume of the sintered pellet. (It is always close to the nominal $\mathrm{MgB}_{2}$ content times 0.7 ). The real volume fraction of $\mathrm{MgB}_{2}$ might be smaller, if $\mathrm{MgB}_{2}$ partly decomposes or reacts with $\mathrm{MgO}$ during sintering. However, the volume fraction used in the following always refers to this calculation.

The temperature dependence of the ac-susceptibility was measured in a commercial SQUID magnetometer (Quantum Design), with an amplitude of $30 \mu \mathrm{T}$. The demagnetization factor of each sample geometry (typical dimensions: $2 \times 2 \times 1.5 \mathrm{~mm}^{3}$ ) was calculated numerically (between 0.45 and 0.65 ) in order to determine the susceptibility correctly.

The resistivity was monitored at various fixed applied fields between 0 and $15 \mathrm{~T}$, while ramping the temperature down at $10 \mathrm{~K} / \mathrm{h}$. Current and voltage contacts were made with 
silver paste. An electric field of around $15 \mu \mathrm{V} / \mathrm{cm}(\max 25, \min 5 \mu \mathrm{V} / \mathrm{cm})$ was applied at room temperature to ensure that the (local) current density within the current path remains comparable for all $\mathrm{MgO}$ contents. This procedure is based on the assumption that the intrinsic resistivity of the conducting material $\left(\mathrm{MgB}_{2}\right), \rho$, does not change and that the increase in resistance results only from the reduction in cross section of the actual current path. The (local) current density $J$ is then only given by the electric field $E(\vec{E}=$ $\rho \vec{J})$. The current was then kept constant (100 nA to $10 \mathrm{~mA})$ during the measurement. The corresponding (local) current density can be estimated to be around $7 \times 10^{3} \mathrm{~A} \mathrm{~m}^{-2}$. A linear fit to $\rho(T)$ was made in the range $0.5 \rho_{n}<\rho<0.9 \rho_{n}$. Extrapolation to the normal state resistivity $\rho_{n}(T)$ defined the (onset) transition temperature $T_{c}$. $\rho_{n}(T)$ was also obtained by linear extrapolation of the normal state resistivity above the transition to lower temperatures. The temperature, where the resistivity apparently reduces to zero (zero resistivity temperature), $T_{\rho=0}$, was evaluated. The upper critical field and the zero resistivity field were obtained by inversion of $T_{c}(B)$ and $T_{\rho=0}(B)$, respectively.

Magnetization loops were recorded in a commercial Vibrating Sample Magnetometer (VSM) at 5 and $20 \mathrm{~K}$. The field was ramped with $0.25 \mathrm{~T} / \mathrm{min}$ from $-2 \mathrm{~T}$ to $5 \mathrm{~T}$ and back to zero. The critical current density was calculated from the irreversible component of the magnetic moment using the Bean model [18]. A self field correction was made numerically [19].

A small anisotropy of the properties was detected in the magnetization measurements. The zero resistivity field is lower by about $3 \%$ in the pure $\mathrm{MgB}_{2}$ sample when the field is oriented parallel to the direction of the uniaxial pressure, than after rotation by $90^{\circ}$. This means that the induced texture is very weak and can be neglected in the following. The self field critical current density is higher by about $75 \%$ in the parallel orientation. This significant difference is possibly caused by small cracks. Large cracks were visible in some pellets, which were perpendicular to the direction of the pressure (at least in the center of the pellet, from where the samples were cut). This is illustrated in Fig. 19. No samples with large visible cracks were used in the measurements, but it is not unlikely that small cracks were present in all samples, which reduce currents flowing perpendicular to the crack direction (Fig. 1k). Only in magnetic fields parallel to the former direction of the pressure, the induced shielding currents always flow parallel to the cracks, which does not result in a suppression of $J_{\mathrm{c}}$ (Fig. 1 $\mathrm{b}$ ). 

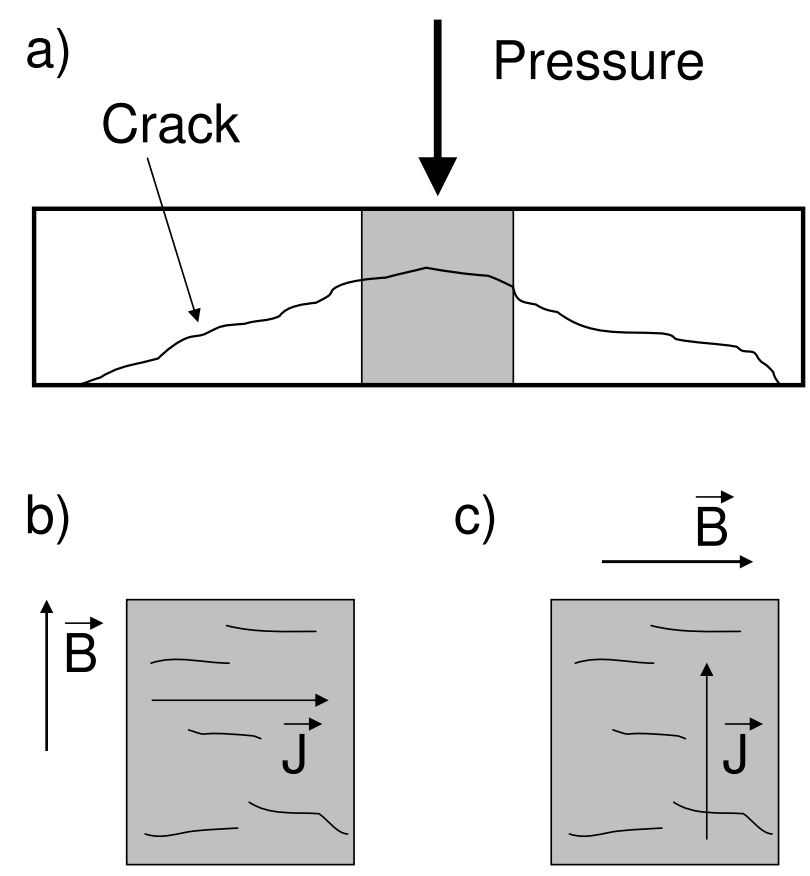

FIG. 1: Large crack in the pellet after pressing (a). Samples for measurements were cut from the center of the pellet (gray area). Orientation of the small cracks, the magnetic field and the currents during resistive (b) and magnetization (c) measurements

The magnetization measurements of all other samples were performed only for one orientation, i.e. with the smallest dimension parallel to the applied field. The field was always perpendicular to the direction of the pressure (Fig. 11). In order to check for possible influences of the geometry of the specimen on the derived current densities, the pure $\mathrm{MgB}_{2}$ sample was also measured with the smallest dimension (and the direction of pressure, which was the longest dimension) perpendicular to the applied field. Nearly identical results were obtained in this case.

The current was applied perpendicular and the field parallel to the direction of the pressure during the resistivity measurements (Fig. 1b).

\section{RESULTS AND DISCUSSION}

The ac susceptibility of samples with different $\mathrm{MgB}_{2}$ content is plotted in Fig. 2, The labels refer to the nominal $\mathrm{MgB}_{2}$ content. The samples containing 0 and $10 \mathrm{vol} \% \mathrm{MgO}$ nearly $(-0.925)$ reach the ideal value, -1 , for complete flux expulsion at low temperatures. The 


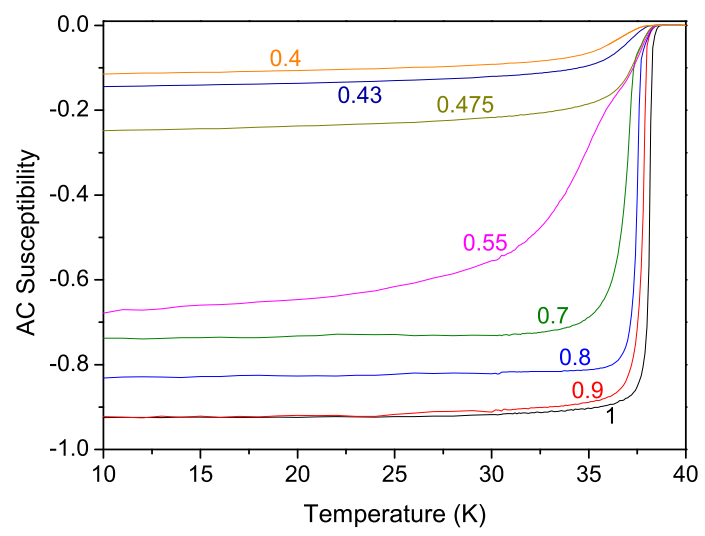

FIG. 2: Change of the ac susceptibility with the nominal $\mathrm{MgB}_{2}$ content, which is given by the labels.

slightly smaller value could result from an overestimation of each dimension by only $2.5 \%$, or by the imperfect geometry of the sample (the numerical calculation of the demagnetization factor is based on a perfect cuboid). The low temperature susceptibility then decreases continuously with $\mathrm{MgB}_{2}$ content, down to $55 \% \mathrm{MgB}_{2}$. This is the first sample with a quite significant broadening of the transition and a non constant susceptibility below $25 \mathrm{~K}$. The further decrease in $\mathrm{MgB}_{2}$ content led to an abrupt reduction of the susceptibility. The samples with 55 and $47.5 \% \mathrm{MgB}_{2}$ behave quite similarly above $37 \mathrm{~K}$, but the susceptibility differs by a factor of about 2.7 at low temperatures. Thus, macroscopic shielding was established in the $55 \%$ sample at low temperatures, while for lower $\mathrm{MgB}_{2}$ contents the length scale of shielding obviously changes. Note that all corresponding transport samples were macroscopically superconducting (zero resistivity), only the $40 \% \mathrm{MgB}_{2}$ sample was apparently insulating.

The superconducting transition temperature, $T_{\mathrm{c}}$, continuously decreases with $\mathrm{MgB}_{2}$ content, although the maximum shift is only about $0.5 \mathrm{~K}$ near the onset of the transition (first points deviating from zero). A larger reduction of $T_{\mathrm{c}}$ was observed at the onset of the resistive transition: from $38.7 \mathrm{~K}$ to $37.8 \mathrm{~K}$, for $100 \%$ and $46 \% \mathrm{MgB}_{2}$, respectively. Zero resistivity was observed at $38 \mathrm{~K}$ and $34.6 \mathrm{~K}$. The residual resistivity ratio decreased from 3.7 in the pure to 1.9 in the $46 \%$ sample and the normalized resistivity $\rho_{\text {norm }}:=\rho(40 \mathrm{~K}) / \Delta \rho$ increased by a factor of 3 . The phonon contribution to the resistivity, $\Delta \rho:=\rho(300 \mathrm{~K})-\rho(40 \mathrm{~K})$, is 


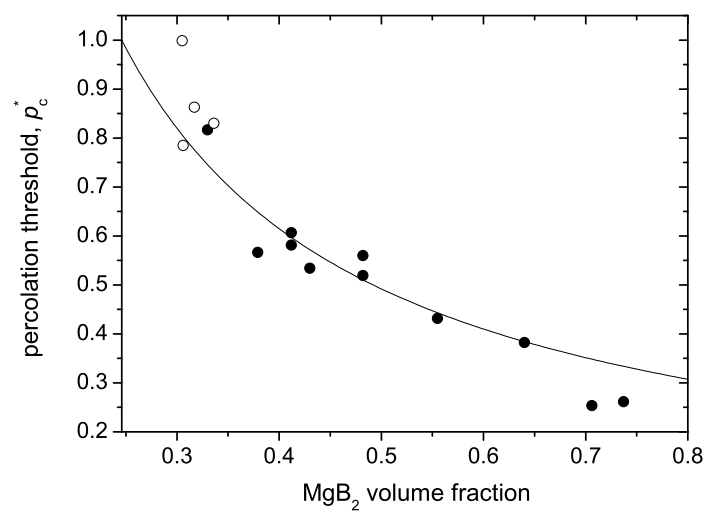

FIG. 3: The percolation threshold as a function of the volume fraction of $\mathrm{MgB}_{2}, p_{\mathrm{MgB}_{2}}$. The open symbols represent data from samples with finite resistivity in the superconducting state.

expected to change hardly with disorder [1], thus the increase in $\rho_{\text {norm }}$ reflects the enhancement of the residual resistivity, $\rho_{0}(\sim \rho(40 \mathrm{~K})$ in the present samples $)$. A linear dependence of $T_{\mathrm{c}}$ on $\rho_{\text {norm }}$ is observed, similar as in previous reports [1, 20, 21, 22]. The corresponding increase in upper critical field is observed in the present samples, too. The changes indicate that the intrinsic properties of the $\mathrm{MgB}_{2}$ grains changed somewhat, either by a slight loss of magnesium, or by any reaction between the two different powders. In any case impurity scattering is increased, which complicates the situation, since not only the volume fraction of $\mathrm{MgB}_{2}$ is varied. In particular the upper critical field anisotropy, $\gamma$, is needed in the following. It is known to be sensitive to impurity scattering [23], but it can be estimated from $T_{\mathrm{c}}$ by [1]

$$
\gamma\left(T_{\mathrm{c}}\right)=\frac{t_{\mathrm{c}}^{2}+16.7 t_{\mathrm{c}}\left(1-t_{\mathrm{c}}\right)}{3.88-3.724 t_{\mathrm{c}}}
$$

with $t_{c}:=T_{\mathrm{c}} / T_{\mathrm{c} 0}$ and $T_{\mathrm{c} 0}=39.43 \mathrm{~K}$.

The percolation cross section $p_{\mathrm{c}}^{*}$ can be estimated from the ratio between the upper critical field and the zero resistivity field, if $\gamma$ is known [12]

$$
p_{\mathrm{c}}^{*}=\sqrt{\frac{\frac{B_{c 2}^{2}}{{\frac{B_{\rho}^{2}}{2}}_{2}^{2}-1}}{\gamma^{2}-1}}
$$

For highly diluted $\mathrm{MgB}_{2}$, when $p_{\mathrm{c}}^{*}$ is close to $1, \gamma$ approaches $B_{\mathrm{c} 2} / B_{\rho=0}$. The highest value for this ratio was 4.75, as found in the sample with the highest, but still finite resistivity (44\% $\left.\mathrm{MgB}_{2}\right)$. Exactly the same value for the anisotropy is obtained from Equ. 3 with the midpoint 
$T_{\mathrm{c}}$ (defined as the temperature, where the resistivity drops to $0.5 \rho_{0}$ ). The midpoint $T_{\mathrm{c}}$ is a reasonable criterion, since it accounts for material inhomogeneities. In this particular sample it was lower by $0.8 \mathrm{~K}$ than the onset $T_{\mathrm{c}}$. This excellent agreement justifies the application of Equ. 3 to estimate $\gamma$ of all samples from the midpoint $T_{\mathrm{c}}$. The percolation threshold $p_{\mathrm{c}}^{*}$ was then calculated from the ratio of the upper critical field and the zero resistivity field (Equ. 4) at $15 \mathrm{~K}$. This temperature was chosen to avoid extrapolation of $B_{\mathrm{c} 2}(T)$ to lower temperatures and $\gamma$ was shown not to change significantly at lower temperatures.

Fig. 3 presents $p_{\mathrm{c}}^{*}$ as a function of the $\mathrm{MgB}_{2}$ volume fraction. The line graph represents the theoretical behaviour $p_{\mathrm{c}}^{*}=p_{\mathrm{c}} / p_{\mathrm{MgB}_{2}}$ with $p_{\mathrm{c}}=0.246$ as obtained by a fit to the solid circles. This value corresponds formally to the percolation threshold of dense pure $\mathrm{MgB}_{2}$ $\left(p_{\mathrm{MgB}_{2}}=1\right)$. The open circles were neglected, since they represent data from samples with a constant but finite resistivity below the superconducting transition in zero field. This indicates either the presence of conducting but not superconducting phases in the remaining highly percolative current path, or normal conducting tunnel currents (see below). With these data points, $p_{\mathrm{c}}$ changes to 0.259 . In the following we assume $p_{\mathrm{c}}=0.25$, which is in between the percolation threshold of a simple cubic lattice $\left(K=6, p_{\mathrm{c}}=0.311\right)$ and a face-centered cubic or hexagonal close packed lattice ( $K=12, p_{\mathrm{c}}=0.199$ in both cases) [9]. It is close to the theoretical $p_{\mathrm{c}}$ of a body-centered cubic lattice $\left(K=8, p_{\mathrm{c}}=0.246\right)$. An average coordination number of 8 seems reasonable in a typical irregular structure.

It should be emphasized that two samples with an $\mathrm{MgB}_{2}$ fraction below 0.3 (but above 0.25) were apparently insulating. This could be due to finite size effects, which are expected to result in a larger percolation threshold, or, simply by the difficulty to make contacts on the fragil spanning cluster, which could be even disrupted during cooling after sintering due to mechanical tensions induced by different thermal expansion coefficients of $\mathrm{MgO}$ and $\mathrm{MgB}_{2}$.

The two points corresponding to pure $\operatorname{MgB}_{2}\left(p_{\mathrm{MgB}_{2}}>0.7\right)$ were not taken into account for the fit in either case, because they obviously deviate from the behaviour of the mixed samples (Fig. 3). The added $\mathrm{MgO}$ powder seems to reduce the average coordination number, possibly due to a different morphology of grains, or by a reaction with $\mathrm{MgB}_{2}$, resulting in a new phase which blocks grain boundaries for supercurrents. The non-zero resistivity of some samples is another hint for the latter scenario. However, extrapolating $p_{\mathrm{c}}^{*}$ of the pure $\mathrm{MgB}_{2}$ samples to the perfectly dense case (again with $p_{\mathrm{c}}^{*}=p_{\mathrm{c}} / p_{\mathrm{MgB}_{2}}$, thus the values of these two 


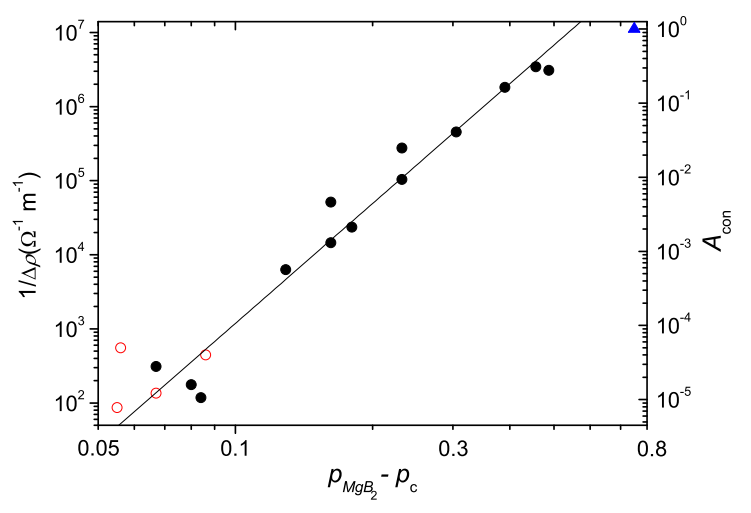

FIG. 4: Change of the conductivity with the $\mathrm{MgB}_{2}$ volume fraction. The line is a fit to the universal behaviour (Equ. 1) with $t=5.4$. The open symbols correspond to samples with finite resistivity in the superconducting state. The triangle in the upper right corner is the expected value for perfect connectivity $(9 \mu \Omega \mathrm{cm})$.

points are multiplied by about 0.7 ), one finds a percolation threshold of about 0.19 , which is low but not unrealistic, since $p_{\mathrm{c}}$ was found to be even lower in some systems [17, 24].

The phonon contribution to the resistivity was introduced as a measure of the effective area in $\mathrm{MgB}_{2}$ [5], since $1 / \Delta \rho$ should be proportional to the cross section over which the current flows, at least if no other conducting phases exist in the sample. $\Delta \rho$ is not expected to be influenced by the observed changes of the superconducting properties.

Fig. 4illustrates that the conductivity can be nicely described by the universal behaviour (Equ. 1). The best approximating (only zero resistivity samples) transport exponent, $t$, is 5.4, which is much higher than the theoretically universal value of $\sim 2$ in three dimensional systems [13]. The transport exponent in disordered continuum systems, which can be described by the so called Swiss-cheese model, is predicted to be only slightly higher, around 2.5 25]. However, larger values were frequently observed experimentally in real systems [17, 24, 26]. The universal behaviour is predicted only for the vicinity of $p_{\mathrm{c}}$ (which is not accessible to the present experiment), thus the application to rather high fractions, $\mathrm{p}$, is problematic and might result in higher transport exponents. As shown recently [27], the transport exponent retains its universal value only very close to $p_{\mathrm{c}}$, if tunnel currents are taken into account and much higher apparent values are expected at higher fractions $p$. 


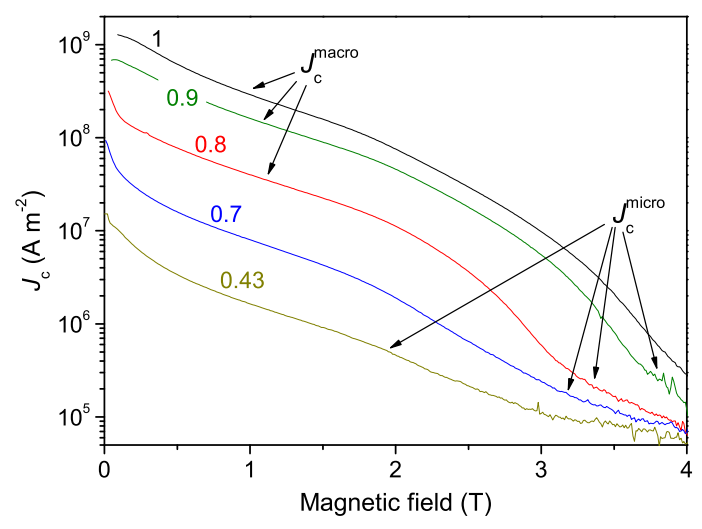

FIG. 5: Influence of the nominal $\mathrm{MgB}_{2}$ content (labels) on the critical current densities at $20 \mathrm{~K}$.

One might speculate, that connections, which are blocked by small $\mathrm{MgO}$ particles or layers, might be transparent for tunneling currents leading to this high transport exponent. This could also explain the finite resistivity of some samples well below $T_{\mathrm{c}}$, if the local current exceeds the maximum Josephson current.

Yamamoto et al. [15] found a larger percolation threshold of 0.31 , but a much weaker dependence of $\Delta \rho$ (and $J_{c}$ ) on the volume fraction of $\mathrm{MgB}_{2}$, which was varied between 0.44 and 0.87 by different preparation techniques (all samples were prepared in-situ). Their nonsuperconducting volume consisted mainly of pores. Thus, it is obvious that pores and $\mathrm{MgO}$ particles have a different influence on the properties of $\mathrm{MgB}_{2}$. This is also supported by the present data, since extrapolation of the data to $p_{\mathrm{MgB}_{2}}=1$ does not lead to the expected value of $\Delta \rho \sim 9 \mu \Omega \mathrm{cm}$ (triangle in Fig. 4), but to $1.67 \mu \Omega \mathrm{cm}$, although this discrepancy could result also from a change in behaviour near $p_{\mathrm{MgB}_{2}}=1$. However, we also found a smaller extrapolated $p_{\mathrm{c}}$ in the pure $\mathrm{MgB}_{2}$ samples (see above).

The critical current densities at $20 \mathrm{~K}$ are presented in Fig. 5, $J_{c}$ at self field is around $10^{9} \mathrm{Am}^{-2}$ in the pure sample, thus considerably smaller than in typical in-situ prepared samples. $J_{c}$ in a sample of comparable connectivity ( $\Delta \rho$ was $29 \mu \Omega \mathrm{cm}$ in our pure sample) was found to be around 4 times higher in Ref. [15]. This can be explained by the different grain size. The grain size of in-situ prepared samples is generally smaller (e.g. 100 nm [15]) than in ex-situ samples (e.g. up to $1 \mu \mathrm{m}[6]$ ). In particular our long heat treatment favors grain growth. Thus, the density of grain boundaries, which are the dominant pinning centers 
in $\mathrm{MgB}_{2}$, is expected to be low in the present samples.

The critical current densities decrease with $\mathrm{MgB}_{2}$ content. The steep slope at higher fields (e.g. between 2 and $3 \mathrm{~T}$ in sample 0.8 ) gradually disappears. This strong decrease in $J_{\mathrm{c}}$ can be associated with the fast reduction in effective cross section (for high currents). At higher fields, after the decomposition of the spanning cluster, $J_{c}(B)$ becomes flatter again, but the currents are now flowing on a different length scale, namely on disconnected clusters of grains. Note that the evaluation of $J_{\mathrm{c}}$ assumes the currents to flow macroscopically around the sample. One obtains the corresponding macroscopic current density $J_{c}^{\text {macro }}$, if this condition is fulfilled. The evaluation becomes invalid if the currents, which flow on a smaller length scale, $J_{\mathrm{c}}^{\text {micro }}$, generate a significant contribution to the total magnetic moment [28]. These additional currents lead to an overestimation of the macroscopic critical current density, because they increase the measured moment. On the other hand, $J_{\mathrm{c}}^{\text {micro }}$ is underestimated, if the macroscopic currents become small or even zero, because the area enclosed by the microcurrents is much smaller than the sample geometry and a priori unknown. Only if the macroscopic currents dominate, the correct $J_{\mathrm{c}}^{\text {macro }}$ is obtained, in all other case the derived magnetic $J_{\mathrm{c}}$ is wrong. Nevertheless, this magnetic $J_{\mathrm{c}}$ illustrates the differences between samples with and without large macroscopic currents. Sample 0.43 was apparently insulating, thus the spanning cluster is very weak or not existing at all, even at zero field. Currents flowing on a microscopic length scale are expected under all conditions (when superconducting grains exist), but it depends on the field and the amount of $\mathrm{MgB}_{2}$ whether they give a negligible (small fields and large $\mathrm{MgB}_{2}$ concentration), important, dominant or even the only contribution (small $\mathrm{MgB}_{2}$ concentration or large fields) to the signal. Their importance generally increases with increasing field and decreasing $\mathrm{MgB}_{2}$ concentration. The slope of $J_{\mathrm{c}}(B)$ is similar in all samples up to about $2 \mathrm{~T}$ (except in the low field region, where geometry dependent self field effects become important), since the length scale of current flow does not change (Fig. [5). The decomposition of the spanning cluster, if existing, always starts near $B_{\mathrm{c} 2} / \gamma$. In principle the weakening of the spanning cluster starts exactly at $B_{\mathrm{c} 2} / \gamma$, where the number of superconducting grains $p$ starts to decrease, but if $p$ is assumed to depend on $J$ [2] ( $p$ decreases with $J$ ) the "spanning cluster for high currents" decomposes at smaller fields. After the destruction of the spanning cluster, the average size and number of the disconnected clusters further depend on $p_{\mathrm{MgB}_{2}} p(B, J)$, leading to different slopes of the magnetic $J_{\mathrm{c}}$ at high fields. Note that $B_{\rho=0}$ derived from resistive measurements falls 


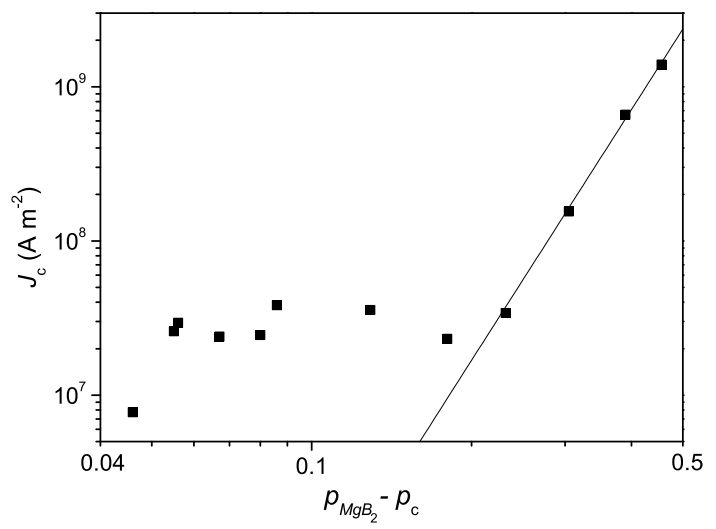

FIG. 6: Critical current densities at $5 \mathrm{~K}$ and $0.5 \mathrm{~T}$. $p_{\mathrm{c}}$ was assumed to be 0.25 .

into this region, e.g. $4.5 \mathrm{~T}$ in sample 0.8 . The influence of the macroscopic currents in the remaining spanning cluster on the magnetization is small, they might be even described by a network of tunneling junctions [5].

The derived critical currents are a useful approximation for the macroscopic currents at low $\mathrm{MgO}$ concentrations and low magnetic fields. $J_{\mathrm{c}}(0.5 \mathrm{~T}, 5 \mathrm{~K})$ is plotted as function of the $\mathrm{MgB}_{2}$ volume fraction (reduced by $p_{\mathrm{c}}=0.25$ ) in Fig. 6. The transport exponent $t=5.4$ is illustrated by the line graph and describes the data very well, down to a volume fraction of about 0.5 . The influence of the microscopically circulating currents becomes dominant at lower concentrations. The observation of the same reduction in cross section for normal and supercurrents, which was also found for porous samples [15], supports the idea of using $\Delta \rho$ to estimate the reduction in $J_{\mathrm{c}}$ due to a reduced connectivity.

\section{CONCLUSIONS}

The percolative current transport was investigated in mixed $\mathrm{MgB}_{2} / \mathrm{MgO}$ samples. The susceptibility, the critical current, the conductivity, and the superconducting transition width increase with $\mathrm{MgO}$ content. The expected change in the relevant length scale for shielding currents was observed in magnetization measurements. An unusually high transport exponent of 5.4 and a percolation threshold of about 0.25 were found. The latter is higher than in porous $\mathrm{MgB}_{2}\left(p_{\mathrm{c}}<0.2\right)$, which has a smaller dependence of the transport 
properties on the superconducting volume fraction than the mixed $\mathrm{MgB}_{2} / \mathrm{MgO}$ samples. A reduction in $J_{\mathrm{c}}$ by a factor of 2 can be expected by the presence of only $10 \% \mathrm{MgO}$.

The phonon contribution to the resistivity was confirmed to be a useful measure for the decrease in $J_{\mathrm{c}}$ due to poor connectivity or density.

\section{Acknowledgments}

The authors wish to thank Herbert Hartmann for technical assistance.

[1] Eisterer M 2007 Supercond. Sci. Technol. 20 R47

[2] Eisterer M, Zehetmayer M and Weber H W 2003 Phys. Rev. Lett. 90247002

[3] Herrmann M, Haessler W, Rodig C, Gruner W, Holzapfel B and Schultz Ludwig 2007 Appl. Phys. Lett. 91082507

[4] Hur J M, Togano K, Matsumoto A, Kumakura H, Wada H and Kimura K 2008 Supercond. Sci. Technol. 21032001

[5] Rowell J M 2003 Supercond. Sci. Technol. 16 R17

[6] Birajdar B, Peranio N and Eibl O 2008 Supercond. Sci. Technol. 21073001

[7] Jiang J, Senkowicz B J, Larbalestier D C and Hellstrom E E 2006 Supercond. Sci. Technol. 19 L33

[8] Klie R F, Idrobo J C, Browning N D, Regan K A, Rogado N S and Cava R J 2001 Appl. Phys. Lett. 791837

[9] van der Marck S C 1997 Phys. Rev. E 551514

[10] Wierman J C and Naor D P 2005 Phys. Rev. B 71036143

[11] Eisterer M, Schöppl K R, Weber H W, Sumption M D. and Bhatia M 2007 IEEE Trans. Appl. Supercond. 172814

[12] Eisterer M, Krutzler C, and Weber H W 2005 J. Appl. Phys. 98033906

[13] Stauffer D and Aharony A Introduction to Percolation Theory. Taylor \& Francis, London, 1992.

[14] Rowell J M, Xu S Y, Zeng X H, Pogrebnyakov A V, Li Qi, Xi X X, Redwig J M, Tian W and Pan X. 2003 Appl. Phys. Lett. 83102 
[15] Yamamoto A, Shimoyama J, Kishio K and Matsushita T 2007 Supercond. Sci. Technol. 20 658

[16] Matsushita T, Kiuchi M, Yamamoto A, Shimoyama J-I and Kishio K 2008 Supercond. Sci. Technol. 21015008

[17] Grinenko V, Krasnoperov E P, Stoliarov V A, Bush A A and Mikhajlov B P 2006 Solid State Comm. 138461

[18] Bean C P, 1964 Rev. Mod. Phys. 3631

[19] Wiesinger H P, Sauerzopf F M and Weber H W 1992 Physica C 203121

[20] Eisterer M, Müller R, Schöppl R, Weber H W, Soltanian S and Dou S X 2007 Supercond. Sci. Technol. 20117

[21] Gandikota R et al. 2005 Appl. Phys. Lett. 86012508

[22] Tarantini C et al. 2006 Phys. Rev. B 73134518

[23] Krutzler C, Zehetmayer M, Eisterer M, Weber H W, Zhigadlo N D and Karpinski J 2007 Phys. Rev. B 75224510

[24] Lee S.-I., Song Yi, Noh T W, Chen X-D and Gaines J R 1986 Phys. Rev. B 346719

[25] Halperin B I, Feng S, and Sen P N 1985 Phys. Rev. Lett. 542391

[26] Vionnet-Menot S, Grimaldi C, Maeder T, Strässler S and Ryser P 2005 Phys. Rev. B 71 064201

[27] Johner N, Grimaldi C, Balberg I and Ryser P 2008 Phys. Rev. B 77174204

[28] Horvat J, Yeoh W K, Kim J H and Dou S X 2008 Supercond. Sci. Technol. 21065003 